\title{
Bioequivalence Study Comparing Fixed-Dose Combination of Clopidogrel and Aspirin with Coadministration of Individual Formulations in Chinese Subjects Under Fed Conditions: A Phase I, Open-Label, Randomized, Crossover Study
}

\author{
Yan Li · Jeffrey E. Ming · Fangyuan Kong · Huiqiu Yin · Linlin Zhang • \\ Haihong Bai · Huijuan Liu · Lu Qi · Yu Wang · Fang Xie · Na Yang • \\ Chuan Ping $\cdot$ Yi Li $\cdot$ Liu Chen $\cdot$ Chunyu Han $\cdot J u$ Liu $\cdot$ Xinghe Wang
}

Received: August 1, 2020 / Published online: September 24, 2020

(c) The Author(s) 2020

\section{ABSTRACT}

Introduction: Simultaneous administration of acetylsalicylic acid (ASA) and clopidogrel has demonstrated efficacy in the treatment of acute coronary syndrome. Clopidogrel + ASA in a fixed-dose combination (FDC) provides a pharmaceutical option to enhance adherence to the coadministration of dual antiplatelet therapy (DAPT). Herein, we evaluate the bioequivalence of enteric ASA and clopidogrel in an FDC

Digital Features To view digital features for this article go to https://doi.org/10.6084/m9.figshare.12859856.

Electronic Supplementary Material The online version of this article (https://doi.org/10.1007/s12325020-01486-9) contains supplementary material, which is available to authorized users.

Y. Li · H. Bai · H. Liu - L. Qi · L. Chen - C. Han ·

J. Liu $\cdot$ X. Wang $(\bowtie)$

Phase I Clinical Trial Center, Beijing Shijitan

Hospital, Capital Medical University, Beijing

100038, China

e-mail: wangxh@bjsjth.cn

J. E. Ming

Research and Development, Sanofi, New York, USA

F. Kong · N. Yang $\cdot$ C. Ping $\cdot$ Y. Li

Research and Development, Sanofi, Beijing, China

L. Zhang

Medical, Sanofi, Beijing, China

H. Yin · F. Xie

Medical, Sanofi, Shanghai, China compared with simultaneous administration of the individual formulations.

Methods: This study is a randomized, single-center, open-label, three-sequence, three-period, twotreatment, crossover study conducted in healthy Chinese male and female subjects under fed conditions. Subjects were randomized to receive, in each period, a single dose of (1) a combination tablet containing 75-mg clopidogrel and 100-mg enteric ASA (test formulation) or (2) coadministration of one 75-mg clopidogrel tablet and one 100-mg enteric-coated ASA tablet (reference formulations) under fed conditions. Plasma samples were analyzed for ASA, salicylic acid, clopidogrel, and the clopidogrel metabolite SR26334. For ASA, the reference-scaled average bioequivalence (RSABE) analysis was conducted for $C_{\max }$ of ASA because within-subject standard deviation $\left(\mathrm{SD}_{\mathrm{W}}\right)$ was $\geq 0.294$ for log-transformed $C_{\max }$.

Results: The point estimate (test/reference geometric mean ratio) was between 0.80 and 1.25 , and the upper one-sided 95\% confidence interval (CI) for the scaled average bioequivalence metric was $\leq 0(-0.08)$. AUC of ASA as $\mathrm{SD}_{\mathrm{W}}$ was $<0.294$ for log-transformed $\mathrm{AUC}_{\text {last }}$ and AUC. Estimates of 90\% CIs for log-transformed $\mathrm{AUC}_{\text {last }}$ and AUC ratios were within the bioequivalence range of 0.80 to $1.25(0.98-1.08$ and $1.00-1.10$, respectively). For clopidogrel, the $90 \%$ CIs for the ratios comparing log-transformed $C_{\text {max }}, \mathrm{AUC}_{\text {last}}$, and $\mathrm{AUC}$ ratios of clopidogrel following administration of test versus reference formulation were calculated using the 
$\mathrm{ABE}$ method and were well within the acceptable range of 0.80 to $1.25(1.02-1.12,0.92-0.99$, and 0.92-0.98, respectively).

Conclusion: FDC of ASA and clopidogrel was bioequivalent to the simultaneous administration of the individual formulations in healthy Chinese subjects under fed conditions.

Trial registration: CTR20190376.

Keywords: ASA; Bioequivalence; Clopidogrel; Fixed dose combination; RSABE

\section{Key Summary Points}

Why carry out this study?

Simultaneous administration of acetylsalicylic acid (ASA) and clopidogrel has demonstrated efficacy in the treatment of acute coronary syndrome. Clopidogrel + ASA in a fixed-dose combination (FDC) provides the pharmaceutical option to enhance adherence of the coadministration of dual antiplatelet therapy (DAPT).

The study evaluated the bioequivalence of enteric ASA and clopidogrel in an FDC compared with simultaneous administration of the individual formulations.

\section{What was learned from the study?}

The point estimate (test/reference geometric mean ratio) for ASA was between 0.80 and 1.25 , and the upper one-sided $95 \%$ confidence interval (CI) for the scaled average bioequivalence metric was $\leq 0$ $(-0.08)$. AUC of ASA as $\mathrm{SD}_{\mathrm{W}}$ was $<0.294$ for log-transformed $\mathrm{AUC}_{\text {last }}$ and AUC.

Bioequivalence was also achieved with clopidogrel as the $90 \%$ CIs for geometric mean ratios of clopidogrel $C_{\text {max }}, \mathrm{AUC}_{\text {last, }}$, and AUCs were within the bioequivalence range (0.80-1.25).

FDC of ASA and clopidogrel was bioequivalent to the simultaneous administration of the individual formulations in healthy Chinese subjects under fed conditions.

\section{DIGITAL FEATURES}

This article is published with digital features to facilitate understanding of the article. You can access the digital features on the article's associated Figshare page. To view digital features for this article go to https://doi.org/10.6084/m9. figshare.12859856.

\section{INTRODUCTION}

Dual antiplatelet therapy (DAPT) with acetylsalicylic acid (ASA) and clopidogrel is considered to be the most effective treatment for patients suffering from acute coronary syndrome (ACS) and coronary artery disease [1]. ASA and clopidogrel exhibit complementary mechanisms of action for inhibiting platelet function. While ASA irreversibly inhibits platelet cyclooxygenase-1 (COX-1) and generation of thromboxane $\mathrm{A}_{2}\left(\mathrm{TXA}_{2}\right)$, which is an inducer of platelet aggregation and vasoconstriction [2], clopidogrel, a $\mathrm{P}_{2} \mathrm{Y}_{12}$ adenosine diphosphate (ADP) receptor antagonist, inhibits ADP-induced platelet aggregation through formation of an inactive carboxylic acid metabolite and an active thiol metabolite [3, 4].

However, regimens of more than one drug taken concurrently may lead to poor or incomplete treatment adherence and suboptimal clinical outcomes. Poor adherence to DAPT is an important contributor to cardiovascular mortality and lethal cardiovascular events [5]. Premature discontinuation of DAPT is a strong predictor for the occurrence of stent thrombosis [6]. To overcome this issue of premature cessation of DAPT, several fixed-dose combination (FDC) formulations containing two or more drugs have been developed. The FDC drugs may improve adherence by reducing the pill burden from multiple to a single pill. The findings of a randomized controlled trial (RCT) for atherosclerotic cardiovascular disease have shown that prescription of FDC drugs resulted in better treatment adherence and lower cardiovascular risk compared with prescription of multiple individual drugs [7]. 
Another RCT demonstrated similar results in which FDC significantly improved treatment adherence by $22 \%$ compared with prescription of individual drugs for secondary cardiovascular prevention following myocardial infarction [8].

There are several reasons for considering an FDC strategy for prevention of cardiovascular disease in China. First, there is a high incidence of non-communicable disease in China with $40 \%$ of the patients being treated with multiple combinations of drugs [9]. The spread of the high blood pressure and blood lipidlowering single-pill combination (SPC) is gradually increasing in China, but the antiplatelet drug SPC has a huge gap in use in China. Furthermore, the current treatment for cardiovascular disease shows challenges in terms of low patient compliance and high treatment costs [9]. Medical services accessed by the majority of patients have a low capacity to handle complicated combination treatments that need separate prescriptions [10]. Thus, an FDC containing enteric-coated ASA and clopidogrel provides a relevant pharmaceutical option to enhance better adherence to and compliance with the coadministration of DAPT [11]. The clopidogrel + enteric-coated ASA tablet is a multiple compressed tablet that contains clopidogrel hydrogen sulfate $(75 \mathrm{mg}$ clopidogrel) in the outer layer and ASA $100 \mathrm{mg}$ in the enteric core tablet. This FDC was developed to target patients who have already been receiving both clopidogrel and ASA for the prevention of atherothrombotic events. Three phase III studies (CURE, CLARITY, and COMMIT) [12-15] have been conducted in patients with ACS (including Chinese patients) who received clopidogrel in addition to ASA worldwide, and its effectiveness and safety have been fully demonstrated. However, the bioequivalence between the FDC and simultaneous intake of single drugs in the Chinese population under fed conditions is unknown. Hence, this study was conducted to assess bioequivalence in the Chinese population under fed conditions.

\section{METHODS}

\section{Ethical Considerations}

The study protocol and informed consent to participate were reviewed and approved by the institutional review board of Beijing Shijitan Hospital, China. The study was conducted in accordance with consensus ethics principles derived from international ethics guidelines, including the Declaration of Helsinki, the International Council for Harmonisation (ICH) guidelines for Good Clinical Practice (GCP), and all applicable laws, rules, and regulations.

\section{Subjects}

Healthy Chinese male or female volunteers aged $\geq 18$ years were certified as healthy based on a comprehensive clinical assessment that included detailed medical history, comprehensive physical examination, vital signs, electrocardiogram (ECG), and laboratory parameters. Female subjects of childbearing age were required to have negative results on a pregnancy test, and only those who agreed to use an appropriate method of contraception during the study period were included. The subjects were restricted in use of concomitant medications, tobacco and alcohol, and supplements throughout the study.

\section{Study Design}

A randomized, single-center, open-label, threetreatment, three-period, three-sequence, crossover study (study no. BEQ16000/CTR20190376) with a 10-day washout period between administrations under fed conditions was conducted in 171 healthy Chinese subjects at Beijing Shijitan Hospital, Capital Medical University, China. The 171 subjects were randomized into one of three sequences (Test (T)/Reference (R)/ $\mathrm{R}, \mathrm{R} / \mathrm{T} / \mathrm{R}, \mathrm{R} / \mathrm{R} / \mathrm{T})$. The randomization list consisted of a block size of 6 , with 171 subjects randomly divided into two treatment groups with three periods and three sequences (Fig. 1). 


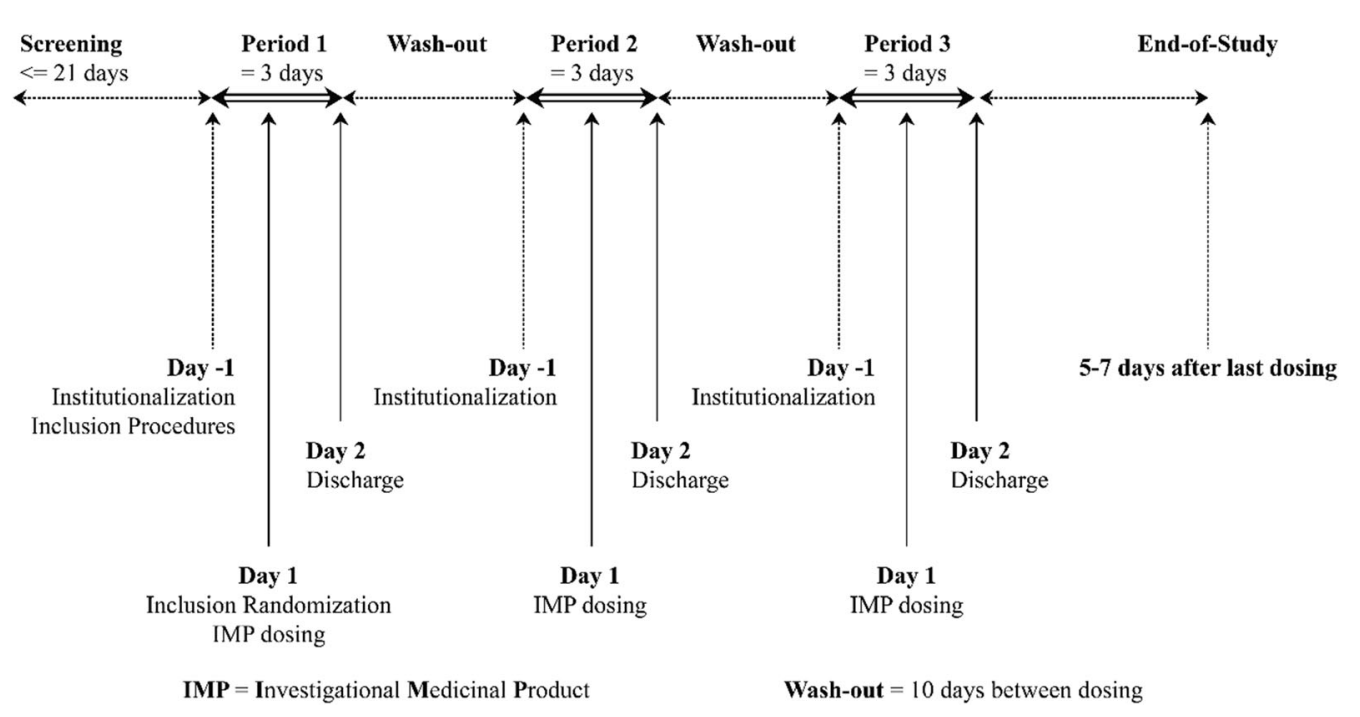

Fig. 1 Study design

\section{Randomization and Interventions}

The test formulation of an FDC containing $75 \mathrm{mg}$ of clopidogrel and $100 \mathrm{mg}$ of enteric ASA was compared with the reference formulations of clopidogrel (Plavix ${ }^{\circledR}, 75 \mathrm{mg}$ ) tablet and enteric-coated ASA (Bayaspirin ${ }^{\circledR}, 100 \mathrm{mg}$ ) tablet. The dose levels for the present study were selected based on the therapeutic doses of Plavix $^{\circledR}$ and Bayaspirin ${ }^{\circledR}$ in China. The subjects were administered the investigational medicinal product (IMP) under fed conditions (standard high-fat breakfast of approximately $1000 \mathrm{kcal}$, with approximately 150, 250, and 500-600 kcal from protein, carbohydrates, and fat, respectively) on day 1 of each period. The subjects were monitored to ensure that their breakfast was completed within $30 \mathrm{~min}$, and then the assigned IMP was administered. No food was allowed for a minimum of $4 \mathrm{~h}$ after administration. On days of treatment administration, standard lunch and dinner were given at least 4 and $10 \mathrm{~h}$ after IMP administration, respectively. The subjects were followed up for 5-7 days after the last administration of IMP.

\section{Objectives}

The primary objective was to determine the bioequivalence of ASA and clopidogrel for an
FDC containing $75 \mathrm{mg}$ of clopidogrel and $100 \mathrm{mg}$ of enteric ASA (test formulation) versus the simultaneous administration of the separate commercial tablets (reference formulations) in healthy Chinese subjects under fed conditions. The secondary objective was to assess the clinical safety of each treatment.

\section{Pharmacokinetic Analysis}

Blood samples were collected at the following time points for ASA/salicylic acid (SA, a metabolite of ASA): $0 \mathrm{~h}$ (pre-dosing) and 2, 3, $3.50,4,4.50,5,5.50,6,6.50,7,7.50,8,10,12$, and $16 \mathrm{~h}$ post-dosing and for clopidogrel/ SR26334: $0 \mathrm{~h}$ (pre-dosing), $0.25,0.50,0.75,1$, $1.50,2,2.50,3,4,5,6,12,16$, and $24 \mathrm{~h}$ postdosing. The parameters assessed were $C_{\max }, t_{\max }$

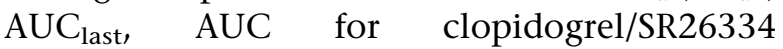
(metabolite of clopidogrel), and ASA/salicylic acid in plasma using non-compartmental methods with Phoenix WinNonlin (Certara USA Inc) version 8.1 (Supplementary Table S1) software. An analysis data set of PK parameters for subjects exposed to a minimum of one dose of IMP formed the Pharmacokinetic Parameter Sets (PKPS), whereas subjects who had a minimum of one evaluable PK parameter from a minimum of one period formed the bioequivalence set (BES). The BES data set was evaluated to determine if the test formulation and the 
reference formulation were bioequivalent. $\mathrm{AUC}_{\text {last }}$ and $\mathrm{t}_{\text {last }}$ were excluded from the BES data set as the sample collection was incomplete. Similarly, the AUC value was excluded from the BES data set as the percentage of AUC determined by extrapolation was $>20 \%$. However, these values were included in the PKPS data set. When a PK profile contained only one quantifiable concentration, $C_{\max }, t_{\max }$, and $t_{\text {last }}$ were excluded from the statistics for BES and PKPS.

\section{Bioanalysis}

The concentrations of clopidogrel/SR26334 and ASA/SA in plasma samples were analyzed using the validated liquid chromatography-tandem mass spectrometry (LC-MS/MS) method. Measurements of clopidogrel and SR26334 were taken by pretreating the plasma samples with liquid-liquid extraction and protein precipitation, respectively. Plasma samples for determination of ASA and salicylic acid concentrations were pretreated by liquid-liquid extraction. The lower limits of quantification (LLOQs) were $5 \mathrm{pg} / \mathrm{ml}$ (clopidogrel), $5 \mathrm{ng} / \mathrm{ml}$ (SR26334), $5 \mathrm{ng} /$ $\mathrm{ml}$ (ASA), and $100 \mathrm{ng} / \mathrm{ml}$ (salicylic acid), and the assays had adequate accuracy and precision in estimating analytes.

\section{Safety Measurements and Analysis}

Subjects were carefully monitored by vital signs, physical examinations, laboratory parameters (hematology, coagulation, biochemistry, and urinalysis), and standard 12-lead ECG. Adverse events were graded according to Common Terminology Criteria for Adverse Events (CTCAE) v4.03 and classified by System Organ Class (SOC) or Preferred Term (PT) according to the latest version of the Medical Dictionary for Regulatory Activities (MedDRA) dictionary.

\section{Statistical Analysis}

\section{Sample Size Determination}

Up to 171 subjects were enrolled to have a minimum of 135 subjects for completion, which was deemed sufficient to provide a $90 \%$ overall power for PK evaluation. For the sample size calculation in this study, the within-subject standard deviation $\left(\mathrm{SD}_{\mathrm{w}}\right)$ of clopidogrel and ASA in fed condition was considered similar to the observed $\mathrm{SD}_{\mathrm{w}}$ in fasting condition based on the completed bioavailability and bioequivalence studies (two published studies and one unpublished study) [16-18].

For clopidogrel, the pooled point estimates of the PK parameter ratios were $0.99,0.98$, and 1.10; the estimates of $\mathrm{SD}_{\mathrm{W}}$ (on the natural log scale) were $0.344,0.335$, and 0.365 for $\mathrm{AUC}_{\text {last, }}$ AUC, and $C_{\text {max }}$, respectively. For ASA, the pooled point estimates of pharmacokinetic (PK) parameter ratios were $1.10,1.10$, and 1.08 , and the estimates of $\mathrm{SD}_{\mathrm{W}}$ (on the natural log scale) were $0.488,0.416$, and 0.696 for $\mathrm{AUC}_{\text {last }}$ AUC, and $C_{\max }$, respectively (the true ratios and true SDw for sample size calculation are presented in Supplementary Table S2). The power of concluding bioequivalence with 135 subjects is provided in Supplementary Table S2. For ASA, the reference-scaled average bioequivalence (RSABE) approach was used, and the standard ABE approach was applied for clopidogrel. The overall power was calculated as a function of the lowest power in clopidogrel and the lowest power in ASA because AUC and $C_{\max }$ were assumed to be highly correlated parameters; therefore, no power loss between AUC and $C_{\max }$ of the same ingredient was considered in the sample size calculation. Hence, the overall power was $90.24 \%(94 \% \times 96 \%)$. In summary, 135 subjects could achieve an overall power of 90\% to conclude bioequivalence between formulations (FDC versus individual formulation) for clopidogrel and ASA. Allowing for a 20\% dropout rate, the total number of subjects for enrollment was 171 .

\section{Bioequivalence Statistical Analysis Plan}

PK parameters of clopidogrel, ASA, and their major metabolites were summarized using descriptive statistics for each formulation. Prior to the analyses, all primary end points $\left(C_{\max }\right.$, $\mathrm{AUC}_{\text {last, }}$ and AUC) were log-transformed. The differences for clopidogrel between the fixeddose formulations and its individual tablet formulation were assessed on log-transformed parameters with a linear mixed-effects model. 
The point estimates and 90\% confidence intervals (CIs) for the geometric mean ratios (GMRs) of $C_{\text {max }}, \mathrm{AUC}_{\text {last}}$, and AUC between the two formulations were obtained within the mixedeffects model framework and then converted to the ratio scale by antilog transformation. Bioequivalence was concluded if the $90 \% \mathrm{CI}$ for the ratio was within $0.80-1.25$.

Bioequivalence for ASA between the FDC and its individual tablet formulation was assessed using the mixed scaled average bioequivalence approach for $C_{\max }, \mathrm{AUC}_{\text {last}}$, and AUC. For each log-transformed parameter, a linear mixedeffects model was formulated, allowing for treatment-specific within-subject variance to obtain the $\mathrm{SD}_{\mathrm{W}}$ for the reference formulation. If $\mathrm{SD}_{\mathrm{W}}$ was $<0.294$, the traditional standard $\mathrm{ABE}$ analysis was conducted within the mixed model framework. Bioequivalence was concluded if the $90 \%$ CI for the formulation ratio (test/reference GMR) was within $0.80-1.25$. If $\mathrm{SD}_{\mathrm{W}}$ was $\geq 0.294$, the RSABE method was conducted as described by Haider et al. [19] (i.e., upper onesided 95\% CI for the RSABE metric). Bioequivalence was concluded if the point estimate (test/ reference GMR) fell within the range of $0.80-1.25$ and the upper one-sided $95 \%$ CI for the RSABE metric was $\leq 0$, (with $\theta=(\ln \Delta)^{2} / \sigma^{2}{ }_{\text {wo }}$ and $\Delta=1.25$, the usual average $\mathrm{BE}$ upper limit for the untransformed test/reference ratio of geometric means, and $\sigma_{\mathrm{w} 0}=0.25$ ) All statistical calculations were performed using Statistical Analysis Software (SAS).

\section{RESULTS}

\section{Subject Demographics}

A total of 171 Chinese healthy male and female subjects recruited between March 2019 and May 2019 were randomized and treated with IMP; 165 subjects completed the study. Six subjects did not complete the study, of whom three subjects discontinued because of adverse events, one subject due to poor compliance to protocol, and two due to withdrawal by the subjects' choice.

There were 111 (64.9\%) healthy male subjects and $60(35.1 \%)$ healthy female subjects included in the study. The mean age of the subjects was 29.8 years, the mean body weight was $64.59 \mathrm{~kg}$, and the mean body mass index was $23.33 \mathrm{~kg} / \mathrm{m}^{2}$. The demographic characteristics are described in detail in Table 1.

\section{Pharmacokinetic Properties}

The mean plasma concentration time profiles for ASA, salicylic acid, clopidogrel, and SR26334 are presented in Figs. 2 and 3. All the PK parameters were similar between the two treatments.

\section{$A S A$}

Systemic exposures to ASA based on $C_{\text {max }}$, $\mathrm{AUC}_{\text {last, }}$ and AUC were similar between the two formulations, with respective arithmetic mean values of $693 \mathrm{ng} / \mathrm{ml}, \quad 939 \mathrm{ng} \cdot \mathrm{h} / \mathrm{ml}$, and $965 \mathrm{ng} \cdot \mathrm{h} / \mathrm{ml}$ for the test formulation and $697 \mathrm{ng} / \mathrm{ml}, 923 \mathrm{ng} \cdot \mathrm{h} / \mathrm{ml}$, and $931 \mathrm{ng} \cdot \mathrm{h} / \mathrm{ml}$ for the reference formulation (pooled results; Table 2). ASA reference PK parameter estimates were consistent when compared across replicates and with pooled results. For administration of reference and test formulations under fed conditions in Chinese healthy subjects, ASA reached the maximum plasma concentration in $6.50 \mathrm{~h}$ (median) post-dose for both FDC and Bayaspirin.

\section{Clopidogrel}

Systemic exposures to clopidogrel based on $C_{\text {max }}, \mathrm{AUC}_{\text {last}}$, and $\mathrm{AUC}$ were similar between the two formulations, with respective arithmetic mean values of $4970 \mathrm{pg} / \mathrm{ml}, 9810 \mathrm{pg} \cdot \mathrm{h} /$

Table 1 Demographics and subject characteristics at baseline

\begin{tabular}{ll}
\hline Variable & Values $(\boldsymbol{N}=\mathbf{1 7 0})$ \\
\hline Age $($ years, mean $\pm \mathrm{SD})$ & $29.8 \pm 5.5$ \\
Males $(n,[\%])$ & $111(64.9 \%)$ \\
Weight $(\mathrm{kg}$, mean $\pm \mathrm{SD})$ & $64.59 \pm 7.61$ \\
Height $(\mathrm{cm}$, mean $\pm \mathrm{SD})$ & $166.6 \pm 8.2$ \\
$\mathrm{BMI}\left(\mathrm{kg} / \mathrm{m}^{2}\right.$, mean $\left.\pm \mathrm{SD}\right)$ & $23.23 \pm 1.89$ \\
\hline
\end{tabular}

$B M I$ body mass index, $S D$ standard deviation 

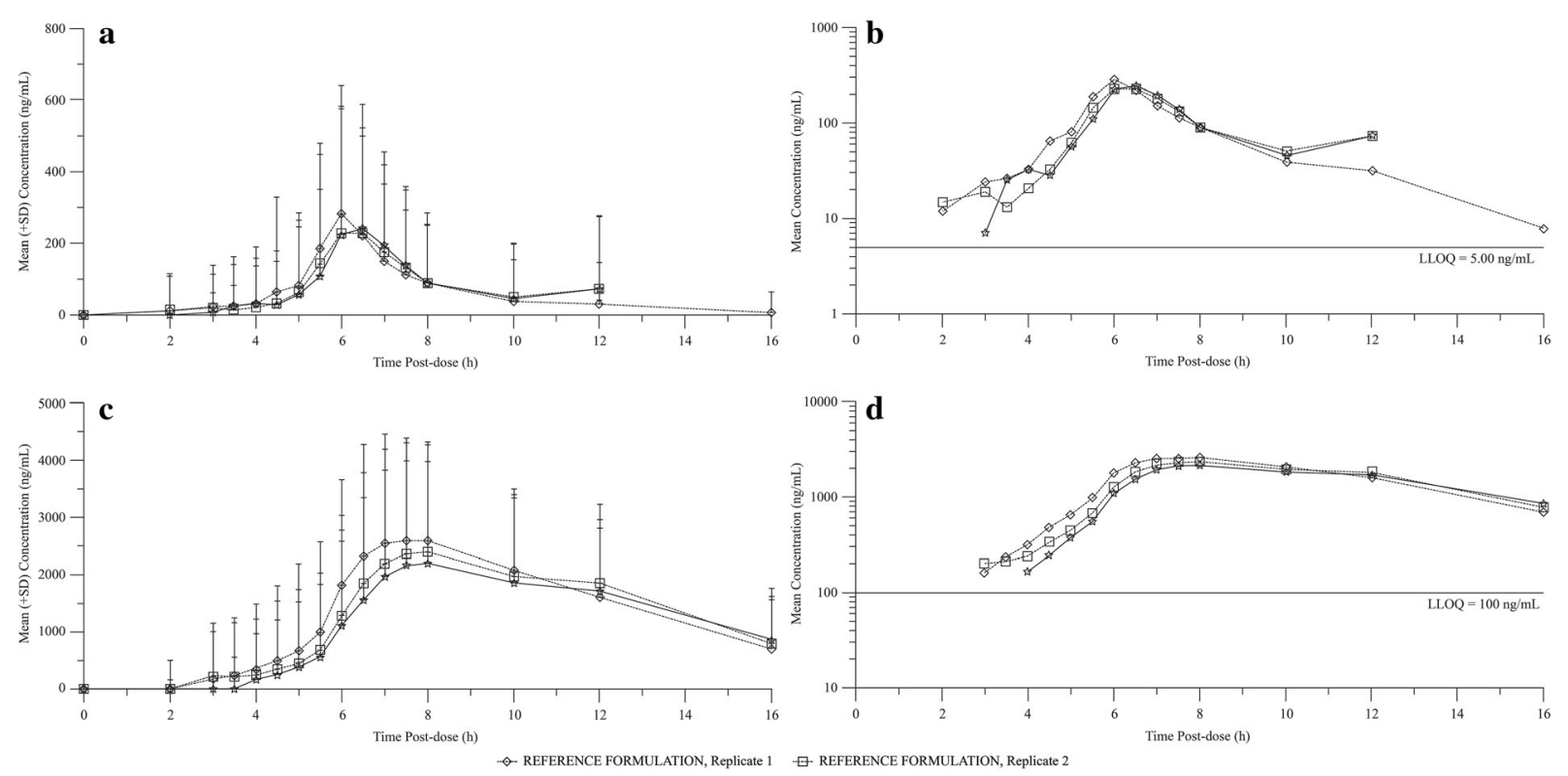
\#- TEST FORMULATION

Fig. 2 Mean plasma concentration versus time profiles of acetylsalicylic acid and salicylic acid following single oral dose administration of test and reference (replicate 1 and 2) formulations to healthy Chinese subjects under fed conditions. a Linear model, ASA; b semi-logarithmic scale, acetylsalicylic acid; c linear model, salicylic acid; $\mathbf{d}$ semilogarithmic scale, salicylic acid
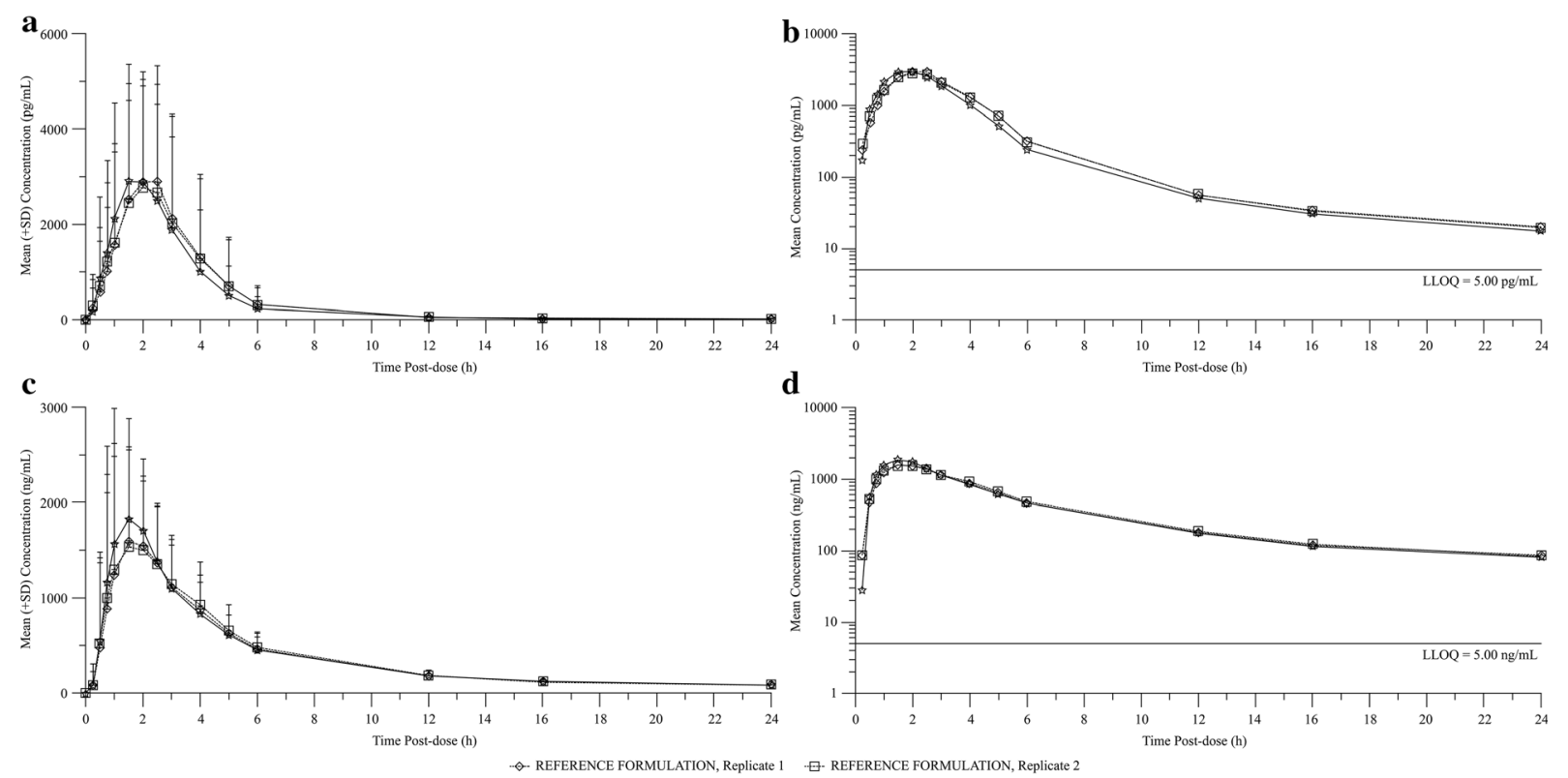

मे TEST FORMULATION,

Fig. 3 Mean plasma concentration versus time profiles of clopidogrel and SR26334 after single oral dose of test and clopidogrel; b semi-logarithmic scale; c linear model, SR26334; d semi-logarithmic scale, SR26334 reference (replicate 1 and 2) formulations to healthy Chinese subjects under fed conditions. a Linear model, 
Table 2 Pharmacokinetic parameters of acetylsalicylic acid and clopidogrel for test formulation (combination tablet) and reference formulation (separate tablets)

\begin{tabular}{|c|c|c|c|c|}
\hline & Combination tablet & Pooled & Replicate 1 & Replicate 2 \\
\hline \multicolumn{5}{|l|}{ Acetylsalicylic acid } \\
\hline \multicolumn{5}{|l|}{$C_{\max }(\mathrm{ng} / \mathrm{ml})$} \\
\hline Number of samples & 145 & 301 & 155 & 146 \\
\hline Mean (SD) & $693(334)$ & $697(340)$ & $704(345)$ & $689(336)$ \\
\hline Geometric mean & 573 & 613 & 631 & 595 \\
\hline CV (\%) & 48 & 49 & 49 & 49 \\
\hline \multicolumn{5}{|l|}{$t_{\max }(\mathrm{h})$} \\
\hline Number of samples & 145 & 301 & 155 & 146 \\
\hline Median & 6.50 & 6.50 & 6.00 & 6.50 \\
\hline Minimum-maximum & $3.00-16.00$ & $2.00-12.00$ & $2.00-12.00$ & $2.00-12.00$ \\
\hline \multicolumn{5}{|l|}{$\mathrm{AUC}_{\text {last }}(\mathrm{ng} \cdot \mathrm{h} / \mathrm{ml})$} \\
\hline Number of samples & 115 & 254 & 135 & 119 \\
\hline Mean (SD) & $939(273)$ & $923(300)$ & $924(318)$ & $921(280)$ \\
\hline Geometric mean & 897 & 878 & 877 & 880 \\
\hline CV (\%) & 29 & 33 & 34 & 30 \\
\hline \multicolumn{5}{|l|}{$\operatorname{AUC}(\mathrm{ng} \cdot \mathrm{h} / \mathrm{ml})$} \\
\hline Number of samples & 95 & 208 & 112 & 96 \\
\hline Mean (SD) & $965(262)$ & $931(278)$ & $928(292)$ & $935(262)$ \\
\hline Geometric mean & 932 & 894 & 889 & 900 \\
\hline CV (\%) & 27 & 30 & 31 & 28 \\
\hline \multicolumn{5}{|l|}{$t_{\text {last }}(\mathrm{h})$} \\
\hline Number of samples & 145 & 300 & 155 & 145 \\
\hline Median & 10.00 & 8.00 & 8.00 & 10.00 \\
\hline Minimum-maximum & $6.00-16.02$ & $5.00-16.02$ & $5.50-16.00$ & $5.00-16.02$ \\
\hline \multicolumn{5}{|l|}{$\mathrm{t}_{1 / 2 \mathrm{z}}(\mathrm{h})$} \\
\hline Number of samples & 95 & 208 & 112 & 96 \\
\hline Mean (SD) & $0.510(0.186)$ & $0.513(0.180)$ & $0.517(0.187)$ & $0.508(0.173)$ \\
\hline Geometric mean & 0.487 & 0.489 & 0.492 & 0.487 \\
\hline CV (\%) & 36 & 35 & 36 & 34 \\
\hline \multicolumn{5}{|l|}{ Clopidogrel } \\
\hline \multicolumn{5}{|l|}{$C_{\max }(\mathrm{pg} / \mathrm{ml})$} \\
\hline Number of samples & 169 & 335 & 169 & 166 \\
\hline
\end{tabular}


Table 2 continued

\begin{tabular}{|c|c|c|c|c|}
\hline & Combination tablet & Pooled & Replicate 1 & Replicate 2 \\
\hline Mean (SD) & $4970(3000)$ & $4670(2890)$ & $4720(3060)$ & $4620(2710)$ \\
\hline Geometric mean & 4350 & 4060 & 4100 & 4030 \\
\hline CV (\%) & 60 & 62 & 65 & 59 \\
\hline \multicolumn{5}{|l|}{$t_{\max }(\mathrm{h})$} \\
\hline Number of samples & 169 & 335 & 169 & 166 \\
\hline Median & 1.50 & 2.00 & 2.00 & 2.00 \\
\hline Minimum-maximum & $0.50-5.00$ & $0.25-5.00$ & $0.25-5.00$ & $0.50-5.00$ \\
\hline \multicolumn{5}{|l|}{$\mathrm{AUC}_{\text {last }}(\mathrm{pg} \cdot \mathrm{h} / \mathrm{ml})$} \\
\hline Number of samples & 169 & 334 & 169 & 165 \\
\hline Mean (SD) & $9810(5950)$ & $10,400(6490)$ & $10,500(6770)$ & $10,200(6220)$ \\
\hline Geometric mean & 8700 & 9110 & 9190 & 9040 \\
\hline CV (\%) & 61 & 63 & 65 & 61 \\
\hline \multicolumn{5}{|l|}{ AUC (pg.h/ml) } \\
\hline Number of samples & 169 & 334 & 168 & 166 \\
\hline Mean (SD) & $10,000(6130)$ & $10,600(6690)$ & $10,800(7000)$ & $10,400(6380)$ \\
\hline Geometric mean & 8880 & 9330 & 9460 & 9210 \\
\hline CV (\%) & 61 & 63 & 65 & 61 \\
\hline \multicolumn{5}{|l|}{$t_{\text {last }}(\mathrm{h})$} \\
\hline Number of samples & 169 & 334 & 169 & 165 \\
\hline Median & 24.00 & 24.00 & 24.00 & 24.00 \\
\hline Minimum-maximum & $16.00-24.02$ & $16.00-24.02$ & $16.00-24.02$ & $16.00-24.02$ \\
\hline \multicolumn{5}{|l|}{$t_{1 / 2 \mathrm{z}}(\mathrm{h})$} \\
\hline Number of samples & 169 & 334 & 168 & 166 \\
\hline Mean (SD) & $7.77(2.25)$ & $7.82(2.48)$ & $8.11(2.57)$ & $7.52(2.37)$ \\
\hline Geometric mean & 7.38 & 7.42 & 7.69 & 7.15 \\
\hline CV (\%) & 29 & 32 & 32 & 31 \\
\hline
\end{tabular}

$A U C$ area under the plasma concentration versus time curve, $C_{\text {max }}$ maximum plasma concentration observed, $A U C_{\text {last }}$ area under the plasma concentration versus time curve calculated using the trapezoidal method from time zero to the real time, $t_{\max }$ time to reach $C_{\max }$ curve, $t_{\text {last }}$ time corresponding to the last concentration above the limit of quantification, $t_{1 / 2 z}$ terminal half-life associated with the terminal slope, $C V$ coefficient of variation 
Table 3 Determination of bioequivalence test method for acetylsalicylic acid

\begin{tabular}{llll}
\hline Parameter & $\mathbf{S}_{\mathbf{W R}}$ & $\mathbf{C V}_{\mathbf{W R}}$ & RSABE conclusion \\
\hline $\log \left(C_{\max }\right)$ & 0.387 & 0.402 & $\begin{array}{c}\text { RSABE applicable } \\
\left(S_{\text {WR }} \geq 0.294\right)\end{array}$ \\
$\log \left(\right.$ AUC $\left._{\text {last }}\right)$ & 0.243 & 0.246 & $\begin{array}{c}\text { RSABE NOT applicable } \\
\left(S_{\text {WR }}<0.294\right)\end{array}$ \\
Log(AUC) & 0.194 & 0.195 & $\begin{array}{c}\text { RSABE NOT applicable } \\
\left(S_{\text {WR }}<0.294\right)\end{array}$ \\
\hline
\end{tabular}

$\mathrm{CV}_{\mathrm{WR}}$ is calculated for raw $\mathrm{PK}$ parameters (i.e., $C_{\text {max }}$, $\mathrm{AUC}_{\text {last }}$, and $\mathrm{AUC}$ )

$A U C$ area under the plasma concentration versus time curve, $C_{\max }$ maximum plasma concentration observed, $A U C_{\text {last }}$ area under the plasma concentration versus time curve calculated using the trapezoidal method from time zero to the real time

$\mathrm{ml}$, and $10,000 \mathrm{pg} \cdot \mathrm{h} / \mathrm{ml}$ for the test formulation and $\quad 4670 \mathrm{pg} / \mathrm{ml}, \quad 10,400 \mathrm{pg} \cdot \mathrm{h} / \mathrm{ml}$, and $10,600 \mathrm{pg} \cdot \mathrm{h} / \mathrm{ml}$ for the reference formulation (pooled results; Table 2). Clopidogrel reference PK parameter estimates were consistent when compared across replicates and with pooled results. Clopidogrel reached the maximum plasma concentration in 1.50 and $2.00 \mathrm{~h}$ (median) post-dose for FDC and Plavix, respectively.

\section{Bioequivalence Evaluation}

Considering that the within-subject variability for the reference $\left(S_{\mathrm{WR}}\right)$ was $\geq 0.294$ for $\log$ transformed $C_{\max }$ for ASA, the RSABE analysis was conducted to assess $C_{\max }$ bioequivalence. For the $C_{\max }$ of ASA, comparing ratios following administration of test versus reference formulation by RSABE analysis showed that the point estimates fell within 0.80-1.25 and the upper limit of the $95 \%$ CIs of RSABE metric was $\leq 0$ (Table 3). The traditional standard ABE analysis was conducted for $\mathrm{AUC}_{\text {last }}$ and AUC of ASA because $\mathrm{SD}_{\mathrm{W}}$ was $<0.294$ for log-transformed $\mathrm{AUC}_{\text {last }}$ and AUC. The 90\% CIs for the ratios comparing log-transformed $\mathrm{AUC}_{\text {last }}$ and $\mathrm{AUC}$ following administration of the test versus reference formulation were within the acceptable range of $0.80-1.25$ for bioequivalence (Table 4). The $90 \%$ CIs for the GMRs of $C_{\max }$, $\mathrm{AUC}_{\text {last, }}$ and $\mathrm{AUC}$ of clopidogrel following administration of test versus reference formulation were well within the acceptable range of 0.80-1.25 (Table 5).

\section{Safety Evaluation}

Of the 171 subjects enrolled, 169 subjects were exposed to both test and reference formulations. No serious adverse events or severe treatment-emergent adverse events (TEAEs) were reported. Of the 10 TEAEs, one subject $(0.6 \%)$ reported one TEAE while receiving the test formulation (diarrhea of grade I intensity), and 9 subjects (5.3\%) reported 14 TEAEs when treated with the reference formulation. One person receiving the reference formulation discontinued treatment because of a TEAE (upper respiratory tract infection). The most frequently reported TEAEs were upper respiratory tract

Table 4 Formulation effect on $\mathrm{C}_{\text {max }}, \mathrm{AUC}_{\text {last }}$, and AUC for acetylsalicylic acid

\begin{tabular}{|c|c|c|c|c|}
\hline Comparison & Parameter & Estimate & \multicolumn{2}{|c|}{ Upper $95 \% \mathrm{CL}$ for $\left(\mu_{\mathrm{T}}-\mu_{\mathrm{R}}\right)^{2}-\theta \times \sigma_{\mathrm{WR}}^{2}$} \\
\hline Test versus reference & $C_{\max }$ & 0.95 & -0.08 & \\
\hline Comparison & \multicolumn{2}{|c|}{ Parameter } & Estimate & $90 \% \mathrm{CI}$ \\
\hline Test versus reference & \multicolumn{2}{|c|}{$\mathrm{AUC}_{\text {last }}$} & 1.03 & $(0.98-1.08)$ \\
\hline & \multicolumn{2}{|c|}{ AUC } & 1.05 & $(1.00-1.10)$ \\
\hline
\end{tabular}

$\theta=\left(\ln (1.25) / \sigma_{0}\right)^{2}$ with $\sigma_{0}=0.250$ corresponding to a regulatory constant

$A U C$ area under the plasma concentration versus time curve, $C I$ confidence interval, $C_{\max }$ maximum plasma concentration observed, $A U C_{\text {last }}$ area under the plasma concentration versus time curve calculated using the trapezoidal method from time zero to the real time 
Table 5 Formulation effect on $C_{\max }, \mathrm{AUC}_{\text {last }}$, and AUC for clopidogrel

\begin{tabular}{llll}
\hline Comparison & Parameter & Estimate & $\mathbf{9 0 \%}$ CI \\
\hline Test versus & $C_{\max }$ & 1.07 & $(1.02-1.12)$ \\
reference & $\mathrm{AUC}_{\text {last }}$ & 0.95 & $(0.92-0.99)$ \\
& $\mathrm{AUC}$ & 0.95 & $(0.92-0.98)$ \\
\hline
\end{tabular}

$A U C$ area under the plasma concentration versus time curve, $C I$ confidence interval, $C_{\max }$ maximum plasma concentration observed, $A U C_{\text {last }}$ area under the plasma concentration versus time curve calculated using the trapezoidal method from time zero to the real time

infection, epistaxis, and upper abdominal pain while receiving the reference formulation, each reported by two subjects (1.2\%). Overall, the test and reference formulations were well tolerated (Table 6).

\section{DISCUSSION}

This is the first study to our knowledge that compares the PK of the FDC of ASA and clopidogrel with that of the coadministered individual formulations under fed conditions. The analysis of the PK parameters demonstrated the bioequivalence of the FDC with their individual formulations in healthy Chinese subjects. Thus, it is expected that the FDC would provide the same therapeutic effect as coadministration of the individual drugs and could be given to Chinese patients who are already taking the two separate drugs.

In 2016, the National Medical Products Administration (NMPA) of China released the new bioequivalence guideline [20], which recommends testing under both fasting and fed conditions. Thus, two separate bioequivalence studies were conducted for the FDC, one under fasting and one under fed conditions. The data reported here show bioequivalence under fed conditions in Chinese subjects. Bioequivalence was also demonstrated under fasting conditions in a separate study.

One important reason for the failure to demonstrate bioequivalence in these studies might be due to insufficient sample sizes using
Table 6 Number (\%) of subjects with TEAE(s) by primary system organ class and preferred term: safety population

\begin{tabular}{lll}
\hline $\begin{array}{l}\text { Primary system organ class } \\
\text { Preferred term }[\boldsymbol{n}(\%)]\end{array}$ & $\begin{array}{l}\text { Reference } \\
(\boldsymbol{N}=\mathbf{1 6 9})\end{array}$ & $\begin{array}{l}\text { Test } \\
(\boldsymbol{N}=\mathbf{1 6 9})\end{array}$ \\
\hline Any class & $9(5.3 \%)$ & $1(0.6 \%)$ \\
Infections and infestations & $2(1.2 \%)$ & 0 \\
$\begin{array}{l}\text { Upper respiratory tract } \\
\text { infection }\end{array}$ & $2(1.2 \%)$ & 0 \\
Cardiac disorders & $1(0.6 \%)$ & 0 \\
Palpitations & $1(0.6 \%)$ & 0 \\
Respiratory, thoracic, and & $2(1.2 \%)$ & 0 \\
$\quad$ mediastinal disorders & & \\
Epistaxis & $2(1.2 \%)$ & 0 \\
Gastrointestinal disorders & $4(2.4 \%)$ & $1(0.6 \%)$ \\
Diarrhea & $1(0.6 \%)$ & $1(0.6 \%)$ \\
Abdominal distension & $1(0.6 \%)$ & 0 \\
Upper abdominal pain & $2(1.2 \%)$ & 0 \\
Anal hemorrhage & $1(0.6 \%)$ & 0 \\
Nausea & $1(0.6 \%)$ & 0 \\
Vomiting & $1(0.6 \%)$ & 0 \\
Investigations & $1(0.6 \%)$ & 0 \\
Blood bilirubin increased & $1(0.6 \%)$ & 0 \\
\hline
\end{tabular}

$N$ number of subjects treated within each group, $n$ (\%) number and $\%$ of subjects with at least one TEAE in each category, TEAE treatment-emergent adverse event

the ABE approach for drugs with high intrasubject coefficient of variation, considering that the GMRs of the test to reference formulation were within the range $0.8-1.25$, although the two-sided $90 \%$ CI for the geometric mean did not meet the bioequivalence criterion. In addition, these studies adopted the two-sequence, two-period, crossover study designs and similar bioanalytic methods. A number of publications have addressed the difficulty of establishing bioequivalence of highly variable drugs $[19,21,22]$. Highly variable drugs are those drugs in which $\mathrm{SD}_{\mathrm{w}}$ is $\geq 0.294$ for the $C_{\max }$ and/ 
or AUC. As per international guidelines, including those from the Food and Drug Administration (FDA) and NMPA, the most suitable approach to evaluate bioequivalence of such highly variable drugs is RSABE, which takes into account the intra-subject variability and is comparable with the reference product in replicate trial design $[19,21,23]$. Various trials have assessed the bioequivalence of highly variable drugs using the RSABE method [24-29]. The RSABE method of analysis employs the use of a three-period, three-sequence, crossover or a four-period, two-sequence, crossover study design for evaluating bioequivalence of highly variable drugs [30].

Because of high intra-subject variability of ASA reported in various studies, in order to minimize the sample size and also to account for intra-subject variability, the RSABE approach was proposed in the study reported here for assessing the bioequivalence of ASA within the FDC. This study was designed as a three-period, three-sequence, crossover, and reference-replicate study under fed conditions instead of a four-period, two-sequence design because the latter design may take a longer duration for study completion, subject compliance may be reduced, and there may be higher rates of dropouts and missing values [31].

Although there were no previous publications of bioequivalence studies for entericcoated ASA under fed conditions, four bioequivalence studies have been conducted in healthy volunteers under fasting conditions $[16,17,32,33]$. All of these studies used the ABE approach for determining bioequivalence Three of these studies in Japanese and Korean subjects did not meet the bioequivalence standard for enteric-coated ASA, possibly because of the high variability of the drug $[16,17,32]$. Another study established bioequivalence in Korean men using the average bioequivalence (ABE) approach; however, the PK data for ASA indicated high intra-subject variability [33]. As no data from studies investigating the intra-individual variability of the primary PK parameters of enteric-coated ASA under fed condition have been reported, we assumed that the $\mathrm{SD}_{\mathrm{w}}$ was similar between the fasting and fed conditions. $\mathrm{SD}_{\mathrm{w}}$ was $>$ 0.294 for the $C_{\max }$ of ASA [32-34] under fasting conditions [35]; hence, an RSABE analysis was conducted to assess the bioequivalence of the PK parameters of ASA with high intra-subject variability, which successfully established the bioequivalence of ASA in the ASA/clopidogrel FDC. Thus, the bioequivalence of ASA and clopidogrel FDC to co-administered individual formulations under fed conditions was established.

The present study has several limitations. First, the subjects enrolled in the study were all between 20 and 40 years of age. However, in the real-world setting, the onset of ACS is mostly observed in an older group of patients [36]. Thus, although the bioequivalence of FDC has been established in a younger group of subjects, a definitive demonstration of bioequivalence in the older patient population would require a study in that population. Second, although a standard breakfast was provided, the calories consumed for a given type of breakfast might have slightly varied across the treatment days for three periods, which might have added to the intra-subject variation for the treatments. Third, the data from this study are limited to the Chinese population under fed conditions, and additional studies would be needed to address the PK in other ethnic groups.

\section{CONCLUSION}

The FDC of ASA and clopidogrel was bioequivalent to the simultaneous administration of the individual formulations in healthy Chinese subjects under fed conditions. Clopidogrel and ASA, administered either as a combination tablet or as separate tablets, were safe and well tolerated in this study.

\section{ACKNOWLEDGEMENTS}

The authors would like to acknowledge Haibiao Jiang, R\&D, Sanofi, China, for manuscript writing guidance, Dan Zheng, Medical Communication, Sanofi, China, for publication process coordination, Yucheng Sheng, a former 
Sanofi employee, for the protocol design, Jin Jing, R\&D, Sanofi, China, for statistical supervisions, Yongzhen $\mathrm{Gu}, \mathrm{R} \& \mathrm{D}$, Sanofi, China, for conducting the study, Jun Lu, R\&D, Sanofi, China, and Rui Chen, R\&D, Sanofi, China, for trial oversight, Yingying Han, Yanqiu Huang, and Jingjing Wei, R\&D, Sanofi, China, for CSR writing support, and Dan Wang, R\&D, Sanofi, China, for pharmacokinetic support.

Funding. This study was sponsored by Sanofi. The sponsor also funded the journal's Rapid Service and Open Access Fees.

Authorship. All named authors meet the International Committee of Medical Journal Editors (ICMJE) criteria for authorship for this article, take responsibility for the integrity of the work as a whole, and have given their approval for this version to be published.

Authorship Contributions. The authors contributed to the concept and design of the study, conducting the analysis, and interpretation of the results. All authors reviewed and approved the final version of the manuscript submitted for publication.

Medical Writing and Editorial Assistance. In addition, the authors would like to acknowledge Anwesha Mandal and Dr. G. Kaushik Subramanian, Indegene Pvt. Ltd, India, for assistance in medical editing under the directions of the authors, supported by Sanofi, according to Good Publication Practice guidelines. The authors individually and collectively are responsible for all content and editorial decisions and received no payment from Sanofi directly or indirectly (through a third party) related to the development or presentation of this publication. The authors also extend thanks to all the participants of the study.

Disclosures. Yan Li, Haihong Bai, Huijuan Liu, Lu Qi, Yu Wang, Liu Chen, Chunyu Han, Ju Liu, and Xinghe Wang have nothing to disclose. Jeffrey E. Ming is an employee of Sanofi and may hold stock and/or stock options in the company. Fangyuan Kong, Huiqiu Yin, Linlin
Zhang, Fang Xie, Na Yang, and Yi Li are employees of Sanofi. Chuan Ping is a former employee of Sanofi.

Compliance with Ethics Guidelines. The study protocol and informed consent to participate were reviewed and approved by the institutional review board of Beijing Shijitan Hospital, China. The study was conducted in accordance with consensus ethics principles derived from international ethics guidelines, including the Declaration of Helsinki, the International Council for Harmonisation (ICH) guidelines for Good Clinical Practice (GCP), and all applicable laws, rules, and regulations.

Data Availability. Qualified researchers may request access to patient-level data and related study documents including the clinical study report, study protocol with any amendments, blank case report form, statistical analysis plan, and data set specifications. Patientlevel data will be anonymized, and study documents will be redacted to protect the privacy of trial participants. Further details on Sanofi's data sharing criteria, eligible studies, and process for requesting access can be found at the following website: https://www.clinicalstudy datarequest.com.

Open Access. This article is licensed under a Creative Commons Attribution-NonCommercial 4.0 International License, which permits any non-commercial use, sharing, adaptation, distribution and reproduction in any medium or format, as long as you give appropriate credit to the original author(s) and the source, provide a link to the Creative Commons licence, and indicate if changes were made. The images or other third party material in this article are included in the article's Creative Commons licence, unless indicated otherwise in a credit line to the material. If material is not included in the article's Creative Commons licence and your intended use is not permitted by statutory regulation or exceeds the permitted use, you will need to obtain permission directly from the copyright holder. To view a copy of this licence, visit http:// creativecommons.org/licenses/by-nc/4.0/. 


\section{REFERENCES}

1. Amsterdam EA, Wenger NK, Brindis RG, et al. 2014 AHA/ACC guideline for the management of patients with non-ST-elevation acute coronary syndromes: executive summary. Circulation. 2014;130:2354-94.

2. Jneid H, Bhatt DL, Corti R, Badimon JJ, Fuster V, Francis GS. Aspirin and clopidogrel in acute coronary syndromes: therapeutic insights from the CURE study. Arch Intern Med. 2003;163:1145-53.

3. Kelly RP, Close SL, Farid NA, Winters KJ, Shen L, Natanegara F, Jakubowski JA, Ho M, Walker JR, Small DS. Pharmacokinetics and pharmacodynamics following maintenance doses of prasugrel and clopidogrel in Chinese carriers of CYP2C19 variants. Br J Clin Pharmacol. 2012;73:93-105.

4. Boggon R, van Staa TP, Timmis A, Hemingway H, Ray KK, Begg A, Emmas C, Fox KAA. Clopidogrel discontinuation after acute coronary syndromes: frequency, predictors and associations with death and myocardial infarction-a hospital registry-primary care linked cohort (MINAP-GPRD). Eur Heart J. 2011;32:2376-86.

5. Collet J-P, Aout M, Alantar A, Coriat P, Napoléon B, Thomas D, Trosini-Desert V, Tucas G, Vicaut E, Montalescot G. Real-life management of dual antiplatelet therapy interruption: the REGINA survey. Arch Cardiovasc Dis. 2009;102:697-710.

6. Grines CL, Bonow RO, Casey DE, et al. Prevention of premature discontinuation of dual antiplatelet therapy in patients with coronary artery stents: a science advisory from the American Heart Association, American College of Cardiology, Society for Cardiovascular Angiography and Interventions, American College of Surgeons, and American Dental Association, with representation from the American College of Physicians. J Am Coll Cardiol. 2007;49:734-9.

7. Thom S, Poulter N, Field J, et al. Effects of a fixeddose combination strategy on adherence and risk factors in patients with or at high risk of CVD: the UMPIRE randomized clinical trial. JAMA. 2013;310: 918-29.

8. Castellano JM, Sanz G, Peñalvo JL, et al. A polypill strategy to improve adherence: results from the FOCUS project. J Am Coll Cardiol. 2014;64: 2071-82.

9. Chinese Expert Consensus and Recommendation Group on the Development of Cardiovascular Disease Polypill in China. Consensus recommendation on the development of cardiovascular disease polypill in China. Zhonghua Xin Xue Guan Bing Za Zhi. 2013;41:91-3.

10. Huffman MD, Rao KD, Pichon-Riviere A, et al. A cross-sectional study of the microeconomic impact of cardiovascular disease hospitalization in four low- and middle-income countries. PLoS One. 2011;6:e20821.

11. Deharo P, Quilici J, Bonnet G, Pankert M, Verdier V, Morange P, Alessi M-C, Bonnet J-L, Cuisset T. Fixeddose aspirin-clopidogrel combination enhances compliance to aspirin after acute coronary syndrome. Int J Cardiol. 2014;172:e1-2.

12. Sabatine MS, Cannon CP, Gibson CM, et al. Addition of clopidogrel to aspirin and fibrinolytic therapy for myocardial infarction with ST-segment elevation. N Engl J Med. 2005;352:1179-89.

13. Sabatine MS, Cannon CP, Gibson CM, et al. Effect of clopidogrel pretreatment before percutaneous coronary intervention in patients with ST-elevation myocardial infarction treated with fibrinolytics: the PCI-CLARITY study. JAMA. 2005;294:1224-322.

14. Yusuf S, Zhao F, Mehta SR, Chrolavicius S, Tognoni G, Fox KK, Clopidogrel in Unstable Angina to Prevent Recurrent Events Trial Investigators. Effects of clopidogrel in addition to aspirin in patients with acute coronary syndromes without ST-segment elevation. N Engl J Med. 2001;345:494-502.

15. Chen ZM, Jiang LX, Chen YP, Xie JX, Pan HC, Peto R, Collins R, Liu LS, COMMIT (ClOpidogrel, and Metoprolol in Myocardial Infarction Trial) collaborative group. Addition of clopidogrel to aspirin in 45,852 patients with acute myocardial infarction: randomised placebo-controlled trial. Lancet. 2005;366:1607-21.

16. (2015) Reexamination report. Pharmaceuticals and Medical Devices Agency. 1-29

17. (2013) Examination report. Pharmaceuticals and Medical Devices Agency, The following are the results of the review by the Pharmaceuticals and Medical Devices Agency for the following drugs that have been submitted for approval. 1-18

18. (2010) Abbreviated Style Clinical Study Report. An open-label, randomized, single-dose, two-sequence crossover relative bioavailability study of a tablet containing $75 \mathrm{mg}$ of clopidogrel and $100 \mathrm{mg}$ of aspirin versus the simultaneous administration of the separate formulations of the two drugs in Japanese healthy male subjects STUDY NUMBER: BDR11360. Unpublished 1-68

19. Haidar SH, Davit B, Chen M-L, et al. Bioequivalence approaches for highly variable drugs and drug products. Pharm Res. 2008;25:237-41. 
20. Food and Drug Inspection and Inspection Center of the State Drug Administration. https://www.cfdi. org.cn/resource/news/9744.html. Accessed 21 May 2020

21. Midha KK, Rawson MJ, Hubbard JW. The bioequivalence of highly variable drugs and drug products. Int J Clin Pharmacol Ther. 2005;43: 485-98.

22. Davit BM, Conner DP, Fabian-Fritsch B, Haidar SH, Jiang X, Patel DT, Seo PRH, Suh K, Thompson CL, $\mathrm{Yu}$ LX. Highly variable drugs: observations from bioequivalence data submitted to the FDA for new generic drug applications. AAPS J. 2008;10:148-56.

23. State Food and Drug Administration (SFDA). Center for drug evaluation. Guideline for bioavailability and bioequivalence studies of generic drug products. https://www.cde.org.cn/zdyz.do?method=lar gepage\&id=2066. Accessed $25 \mathrm{Jan} 08$. Google Search. https://www.google.com/search?q=State+ Food+and+Drug+Administration+(SFDA).+Center + for + drug + evaluation. + Guideline + for + bioavaila bility+ and+bioequivalence+studies + of + generic + drug + products. +Available+from $\% 3 \mathrm{~A}+\% \mathrm{E} 2 \% 8 \mathrm{C} \%$ A9http $\% 3 \mathrm{~A} \% 2 \mathrm{~F} \% 2 \mathrm{Fwww.cde}$. org.cn\%2Fzdyz.do\% 3F\%E2\%8C\%AAmethod $\% 3$ Dlarge+page $\% 26$ id $\% 3$ D2066. $+\% 5$ Baccessed $+25.01 .08 \% 5$ D\&rlz $=1 \mathrm{C} 1 \mathrm{GCE}$ U_enIN872IN872\&oq=State+Food+and+Drug+Adm inistration+(SFDA).+Center+for+drug+evaluation. +Guideline+for+bioavailability+and+bioequivalen ce+studies+of+generic+drug+products.+Available + from $\% 3 \mathrm{~A}+\% \mathrm{E} 2 \% 8 \mathrm{C} \% \mathrm{~A} 9 \mathrm{http} \% 3 \mathrm{~A} \% 2 \mathrm{~F} \% 2 \mathrm{Fwww}$. cde.org.cn\%2Fzdyz.do\%3F\%E2\%8C\%AAmethod\% 3Dlarge+page $\% 26$ id $\% 3$ D2066.+\%5Baccessed +25.01 . 08\%5D\&aqs=chrome..69i57\&sourceid=chrome\&ie= UTF-8. Accessed 17 Mar 2020

24. Oh M, Ghim J-L, Park S-E, Kim E-Y, Shin J-G. Pharmacokinetic comparison of a fixed-dose combination versus concomitant administration of fimasartan, amlodipine, and rosuvastatin using partial replicated design in healthy adult subjects. Drug Des Devel Ther. 2018;12:1157-64.

25. Zhang Y, Chen X, Tang Y, Lu Y, Guo L, Zhong D. Bioequivalence of generic alendronate sodium tablets $(70 \mathrm{mg})$ to Fosamax ${ }^{\text {\&reg; }}$ tablets $(70 \mathrm{mg})$ in fasting, healthy volunteers: a randomized, openlabel, three-way, reference-replicated crossover study. Drug Des Dev Ther. 2017. https://doi.org/10. 2147/DDDT.S138286.

26. Pei Q, Wang Y, Hu Z-Y, et al. Evaluation of the highly variable agomelatine pharmacokinetics in Chinese healthy subjects to support bioequivalence study. PLoS One. 2014;9:e109300.
27. Tang F, Zhou R, Cheng Z, et al. Implementation of a reference-scaled average bioequivalence approach for highly variable generic drug products of agomelatine in Chinese subjects. Acta Pharm Sin B. 2016;6:71-8.

28. Xu S-M, Yan J, Li D, Li D, Li X-M, Xu P-S. Implementation of a reference-scaled average bioequivalence approach for highly variable generic drug products of atorvastatin in Chinese subjects. Int J Clin Pharmacol Ther. 2020;58:112-20.

29. Ding YH, Liu B, Lou JF, et al. Bioequivalence of sarpogrelate in healthy Chinese subjects under fasting and fed conditions: a 4-way replicate crossover investigation by a reference-scaled average bioequivalence approach. Clin Pharmacol Drug Dev. 2019;8:713-20.

30. Tothfalusi L, Endrenyi L. An exact procedure for the evaluation of reference-scaled average bioequivalence. AAPS J. 2016;18:476-89.

31. Chow S-C (2015) Quantitative methods for traditional chinese medicine development, 0 ed. https:// doi.org/10.1201/b18946

32. Jung JA, Kim T-E, Kim J-R, Kim M-J, Huh W, Park $\mathrm{K}-\mathrm{M}$, Lee S-Y, Ko J-W. The pharmacokinetics and safety of a fixed-dose combination of acetylsalicylic acid and clopidogrel compared with the concurrent administration of acetylsalicylic acid and clopidogrel in healthy subjects: a randomized, open-label, 2-sequence, 2-period, single-dose crossover study. Clin Ther. 2013;35:985-94.

33. Choi H-K, Ghim J-L, Shon J, Choi Y-K, Jung JA. Pharmacokinetics and relative bioavailability of fixed-dose combination of clopidogrel and aspirin versus coadministration of individual formulations in healthy Korean men. Drug Des Dev Ther. 2016;10:3493-9.

34. Center for Drug Evaluation and Research, Clinical Pharmacology and Biopharmaceutics Review.

35. Mojaverian P, Rocci ML, Conner DP, Abrams WB, Vlasses PH. Effect of food on the absorption of enteric-coated aspirin: correlation with gastric residence time. Clin Pharmacol Ther. 1987;41:11-7.

36. Simms AD, Batin PD, Kurian J, Durham N, Gale CP. Acute coronary syndromes: an old age problem. J Geriatr Cardiol. 2012;9:192-6. 